\title{
Pyogenic granuloma in a patient with psoriasis successfully treated by 5-aminolevulinic acid photodynamic therapy: A case report
}

\author{
JUAN LIU, BING-RONG ZHOU, FEI YI, HONG-JIN WU, JIA-AN ZHANG and DAN LUO
}

Department of Dermatology, The First Affiliated Hospital of Nanjing Medical University, Nanjing, Jiangsu 210029, P.R. China

Received February 4, 2015; Accepted October 13, 2015

DOI: $10.3892 /$ etm.2015.2899

\begin{abstract}
Pyogenic granuloma (PG) is an acquired benign vascular tumor of unknown etiology. In the present case report, PG was detected in a 49-year-old Chinese male patient with chronic plaque psoriasis. The psoriasis lesions on the finger where the granuloma had developed had been scratched excessively, as declared by the patient. No retinoid therapeutic agents were used during treatment. The patient responded poorly to cryotherapy and surgical curettage. However, following one session of 5-aminolevulinic acid photodynamic therapy (ALA-PDT), signs of improvement were demonstrated 1 week after the treatment, and 1 month following treatment, there were no signs of reoccurrence. Although a report demonstrating treatment success in one patient may be inadequate to estimate the true efficiency of ALA-PDT, dermatologists may consider ALA-PDT as an alternative therapy for stubborn PG.
\end{abstract}

\section{Introduction}

Pyogenic granuloma (PG), which is a rapidly growing benign vascular tumor of unknown etiology on the skin and/or subcutaneous tissue, most commonly presents as red or purple smooth papules on the fingers of patients. Furthermore, PGs bleed easily and commonly result after a minor trauma (1). No more than 20 PG cases have been detected in patients with psoriasis (2). Psoriasis is a chronic inflammatory skin disease with a complex etiology, involving various genetic risk factors and environmental triggers, and affecting 2-3\% of the worldwide population (3). Piraccini et al have indicated that certain systemic inflammatory diseases, such as psoriasis,

Correspondence to: Dr Bing-Rong Zhou, Department of Dermatology, The First Affiliated Hospital of Nanjing Medical University, 300 Guangzhou Road, Nanjing, Jiangsu 210029, P.R. China

E-mail: bingrong.2002@163.com

Abbreviations: ALA-PDT; 5-aminolevulinic acid photodynamic therapy; PG, pyogenic granuloma; VEGF, vascular endothelial growth factor

Key words: pyogenic granuloma, ALA-PDT, VEGF, psoriasis may induce granulation tissue, thus predisposing to the development of PGs (1). Histologically, PGs appear as hyperplastic clusters of capillaries, separated by thin fibrous bands arranged in a lobular configuration. Traditional therapeutic options are available, including: Cryotherapy, electrodessication, curettage or shave removal, surgical excision, various laser therapies, micro-embolization and sclerotherapy (4). However, some patients with PG exhibit poor responses to these therapies. In the present study, a patient with psoriasis presented with a single granuloma that bled easily; biopsy results were consistent with a PG that ultimately healed with only one session of 5-aminolevulinic acid photodynamic therapy (ALA-PDT).

\section{Case report}

A 49-year-old Chinese man suffering from chronic plaque psoriasis presented with an easily bleeding granuloma on the fifth digit of his left hand, following repeated attempts to remove the scales present on the psoriasis lesions. Written informed consent for individual details and accompanying images was obtained from the patient prior to publication of the present case report. The patient had a 12-year history of chronic plaque psoriasis, affecting the scalp, trunk, and limbs (Fig. 1A) and for several years was being treated with methotrexate; however, the results were unsatisfactory. A small granulation tissue developed on the fifth digit of the patient's left hand following repeated scratching. Clinical examination demonstrated a single fleshy, hemorrhagic papule (diameter, $\sim 1.5 \mathrm{~cm}$; height, $5 \mathrm{~mm}$ ) and some erosion on the fifth digit of the patient's left hand (Fig. 1B). Fungal and bacterial cultures were negative. Routine laboratory tests, serum chemistry, hematology and urinalysis, and an antinuclear antibody profile demonstrated no abnormal findings.

A biopsy of $\sim 0.5 \mathrm{~cm}^{3}$ skin bleeding granuloma was obtained from the fifth digit of the patient's left hand for histologic evaluation. Serial sections $(4 \mathrm{~mm})$ were mounted onto silane-coated slides and stained using hematoxylin-eosin (KeyGen Biotech. Co. Ltd., Nanjing, China). In addition, microscopic evaluation (Olympus BH2 microscope; Olympus Corp., Tokyo, Japan) was performed and digital images of the slides were captured. Histopathological examination detected a lobulated proliferation of capillary vessels circumscribed in edematous stroma, and a dense mixed perivascular cellular infiltrate confirmed the clinical diagnosis 
A

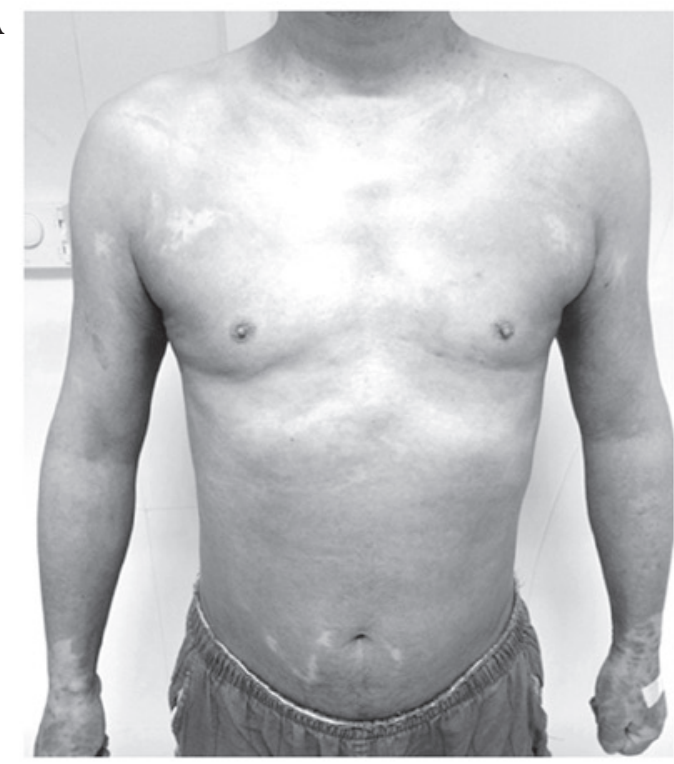

B

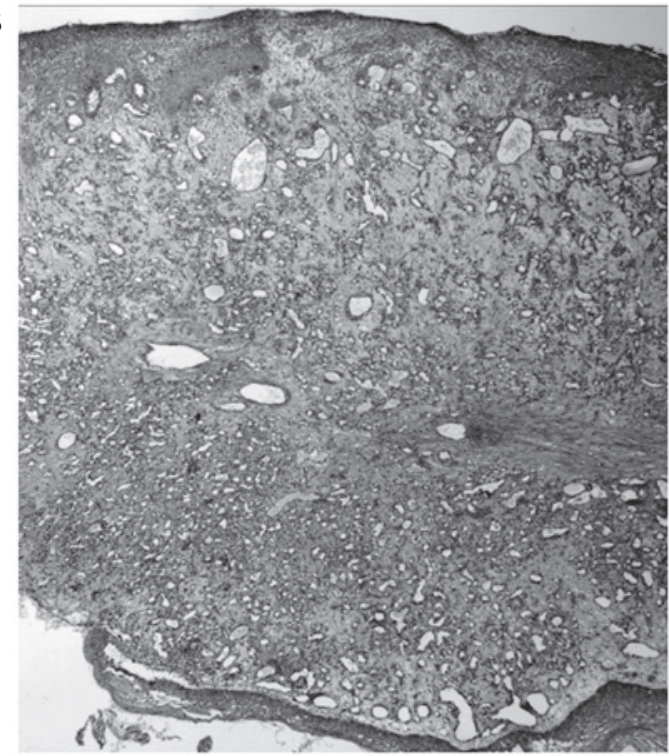

C

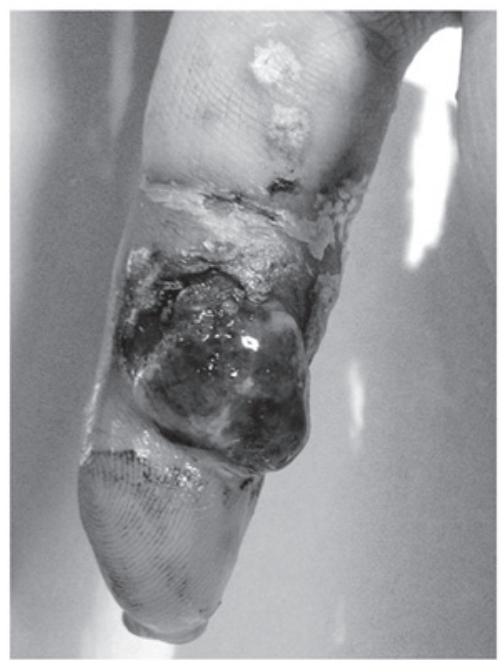

D

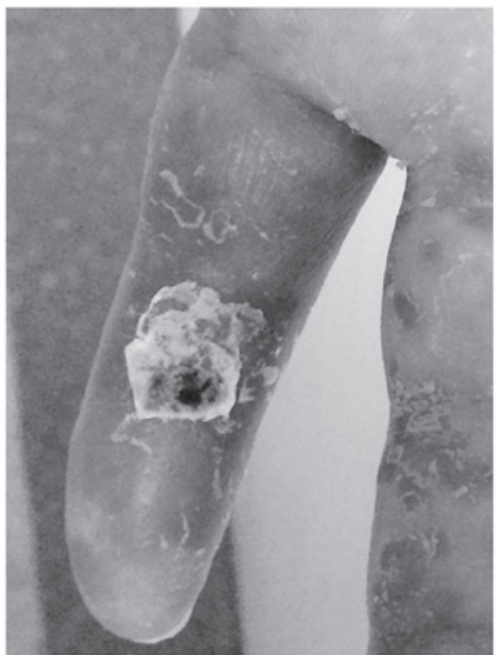

$\mathbf{E}$

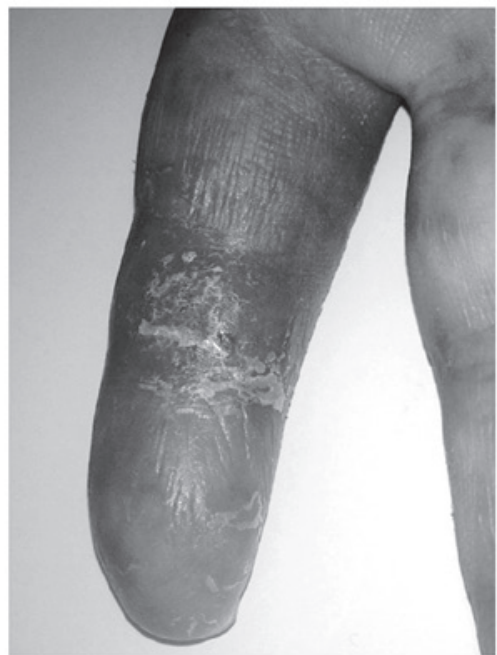

Figure 1. (A) Clinical appearance of psoriasis lesions on the trunk of a 49-year-old Chinese male patient. (B) Histopathological examination following hematoxylin and eosin staining of the granuloma lesion on the fifth digit demonstrated the lobulated proliferation of capillary vessels circumscribed in edematous stroma and a dense mixed perivascular cellular infiltrate (magnification, 10x). (C) Clinical appearance of the granuloma lesions on the fifth digit of the patient's left hand. The clinical appearance of the granuloma lesions on the fifth digit of the patient's left hand (D) 1 week and (E) 1 month following treatment with 5-aminolevulinic acid photodynamic therapy.

of pyogenic granuloma (PG) (Fig. 1C). Cryotherapy was performed twice, however the outcomes were unsatisfactory. Subsequent surgical curettage was performed; however, PG recurred and progressed rapidly 1 week later. Following this, topical ALA-PDT was performed. ALA lotion (20\%; Shanghai Fudan Zhangjiang Bio-Pharmaceutical Co. Ltd., Shanghai, China) was applied onto the area of granulation and occluded with saran wrap for $\sim 3 \mathrm{~h}$. The left fifth digit was then irradiated with narrow band LED $(633 \pm 10 \mathrm{~nm}$; LED-IB; Wuhan Yage Optic and Electronic Technique Co. Ltd., Hubei, China) at a light intensity of $90 \mathrm{~mW} / \mathrm{cm}^{2}$ for $\sim 30 \mathrm{~min}$. The patient received one session of ALA-PDT treatment and was followed-up weekly for 1 month. One week following the ALA-PDT treatment, the erosions had dried up, and the PG lesion was encrusted (Fig. 1D). No signs of recurrence were demonstrated 1 month after treatment (Fig. 1E). The patient did not receive any further treatment during the 1-month follow-up.

\section{Discussion}

A previous study demonstrated that therapeutic agents, local mechanical trauma, peripheral nerve injury and inflammatory systemic diseases are the most frequent causes of PG (1). Furthermore, PG has previously been described as a side-effect of systemic retinoids, such as isotretinoin and etretinate $(5,6)$. However, as no retinoid drugs were used to treat the patient with psoriasis in the present case report, retinoids may not be considered the cause.

The development of PG may reflect the underlying effects of angiogenic factors, such as vascular endothelial growth factor (VEGF), which was overexpressed in psoriasis lesions (7). Activated T cells, inflammatory cells, and keratinocytes in psoriasis lesions (7) are capable of inducing VEGF secretion. The association between the incidence of psoriasis and the serum concentrations of VEGF has previously been confirmed (8). VEGF is recognized as an indicator of disease 
severity (9), and the VEGF/VEGF receptor signaling system has previously been investigated as a potential therapeutic strategy for the treatment of psoriasis (10). Therefore, the hypersecretion of VEGF in psoriasis lesions may provide conditions that promote the development of PG. Previous studies have demonstrated that PG may develop during the treatment of psoriasis $(2,11)$. Frictional PG was initially identified in 2001 (12), outlining that trauma may increase the probability of foreign bodies penetrating wounds in skin tissue, with the subsequent inflammatory reaction leading to the formation of PG (12). The authors of the present case report hypothesize that VEGF was overexpressed in the psoriasis lesions of the patient and foreign bodies were introduced into the wound upon scratching, which subsequently induced an inflammatory reaction and the development of PG.

Various therapeutic strategies have been employed to treat patients with PG. Lee et al (13), demonstrated that surgical excision offered the lowest recurrence rates (2.94\%) among the surgical management options for PG, whereas cryotherapy exhibited the lowest overall recurrence rate (1.62\%). However, the present patient responded poorly to these two first-line treatment options for PG. ALA-PDT employs molecular oxygen, which is a photosensitizing agent, and visible light in order to produce reactive oxygen species that destroy tumor and tumor vasculature cells. ALA-PDT has been widely used to treat skin cancer, acne, warts and various other diseases in the field of dermatology and venereology (14). Furthermore, PDT has previously been used to treat vascular lesions such as port-wine stains and Kaposi's sarcoma $(15,16)$. Lee et al $(17)$ demonstrated that PDT with 5-ALA intralesional injection may be an effective alternative therapeutic technique for the treatment of PG. Previous studies have demonstrated that endothelial cells generate protoporphyrin IX from photosensitizers and PDT has the ability to destroy vascular endothelial cells both in vitro and in vivo $(18,19)$. In addition, VEGF expression was downregulated following ALA-PDT treatment in mice (20). These previous studies may provide evidence regarding the underlying mechanisms of ALA-PDT when used to treat vascular tumors such as PG, as described in the present case. To the best of our knowledge, the present study is the first to report a case of PG that has been successfully treated by topical ALA-PDT.

\section{Acknowledgements}

The present case report was supported by grants from the Priority Academic Program Development of Jiangsu Higher Education Institutions (no. JX10231801) and the Shanghai Fudan-Zhangjiang Bio-Pharmaceutical Corporation (2013).

\section{References}

1. Piraccini BM, Venturi M and Patrizi A: Periungual pyogenic granulomas due to topical tazarotene for nail psoriasis. G Ital Dermatol Venereol 149: 363-366, 2014.

2. Piraccini BM, Bellavista S, Misciali C, Tosti A, de Berker D and Richert B: Periungual and subungual pyogenic granuloma. Br J Dermatol 163: 941-953, 2010.

3. Lowes MA, Bowcock AM and Krueger JG: Pathogenesis and therapy of psoriasis. Nature 445: 866-873, 2007.

4. Millsop JW, Trinh N, Winterfield L, Berrios R, Hutchens KA and Tung R: Resolution of recalcitrant pyogenic granuloma with laser, corticosteroid, and timolol therapy. Dermatol Online J 20, 2014.

5. Campbell JP, Grekin RC, Ellis CN, Matsuda-John SS, Swanson NA and Voorhees JJ: Retinoid therapy is associated with excess granulation tissue responses. J Am Acad Dermatol 9: 708-713, 1983.

6. Badri T, Hawilo AM, Benmously R, Fenniche S and Mokhtar I: Acitretin-induced pyogenic granuloma. Acta Dermatovenerol Alp Pannonica Adriat 20: 217-218, 2011.

7. Das RP, Jain AK and Ramesh V: Current concepts in the pathogenesis of psoriasis. Indian J Dermatol 54: 7-12, 2009.

8. Hugh J, Van Voorhees AS, Nijhawan RI, et al: From the Medical Board of the National Psoriasis Foundation: The risk of cardiovascular disease in individuals with psoriasis and the potential impact of current therapies. J Am Acad Dermatol 70: 168-177, 2014.

9. Flisiak I, Zaniewski P, Rogalska-Taranta M and Chodynicka B: Effect of psoriasis therapy on VEGF and its soluble receptors serum concentrations. J Eur Acad Dermatol Venereol 26: 302-307, 2012.

10. Li W, Man XY, Chen JQ, Zhou J, Cai SQ and Zheng M: Targeting VEGF/VEGFR in the treatment of psoriasis. Discov Med 18: 97-104, 2014.

11. PatrunoC,Balato N,Cirillo T,Napolitano Mand Ayala F: Periungual and subungual pyogenic granuloma following anti-TNF- $\alpha$ therapy: Is it the first case? Dermatol Ther 26: 493-495, 2013.

12. Richert B: Frictional pyogenic granuloma of the nail bed. Dermatology 202: 80-81,2001.

13. Lee J, Sinno H, Tahiri Y and Gilardino MS: Treatment options for cutaneous pyogenic granulomas: A review. J Plast Reconstr Aesthet Surg 64: 1216-1220, 2011.

14. Wan MT and Lin JY: Current evidence and applications of photodynamic therapy in dermatology. Clin Cosmet Investig Dermatol 7: 145-163, 2014.

15. Li W, Yamada I, Masumoto K, Ueda Y and Hashimoto K: Photodynamic therapy with intradermal administration of 5-aminolevulinic acid for port-wine stains. J Dermatolog Treat 21: 232-239, 2010

16. Park MY and Kim YC: Classic Kaposi sarcoma treated with intralesional 5-aminolevulinic acid injection photodynamic therapy. Arch Dermatol 145: 1200-1202, 2009.

17. Lee DJ, Kim EH, Jang YH and Kim YC: Photodynamic therapy with 5-aminolevulinic acid intralesional injection for pyogenic granuloma. Arch Dermatol 148: 126-128, 2012.

18. Rodriguez L, de Bruijn HS, Di Venosa G, Mamone L, Robinson J, Juarranz A, Batlle A and Casas A: Porphyrin synthesis from aminolevulinic acid esters in endothelial cells and its role in photodynamic therapy. J Photochem Photobiol B 96: 249-254, 2009.

19. Chang CJ, Sun CH, Liaw LH, Berns MW and Nelson JS: In vitro and in vivo photosensitizing capabilities of 5-ALA versus photofrin in vascular endothelial cells. Lasers Surg Med 24: 178-186, 1999.

20. Xie Y, Wei ZB, Zhang Z, Wen W and Huang GW: Effect of 5-ALA-PDT on VEGF and PCNA expression in human NPC-bearing nude mice. Oncol Rep 22: 1365-1371, 2009. 